\title{
Study of Partial-Phase Steady-State Operation Modes of Phase-Shift Transformer with Hexagonal Circuit and Regulating Autotransformer
}

\author{
Bosneaga V., Suslov V. \\ Institute of Power Engineering of Moldova \\ Chisinau, Republic of Moldova
}

\begin{abstract}
The scope of the work was the study of the phase failure operating for the new FACT's type phase-shifting device, intended for the flexible connection of AC power systems. The mathamatical model has been developed for conducting this study. The device contains the main phase-shifting transformer based on hexagon circuit with additional regulating autotransformer, this creates the possibility of circular regulation of phase shift angle between connected systems. The model includes two 6-winding three-legs transformers, for which two sets of parameters can be independently set based on the data for the short circuit and no-load modes. The data for the direct sequence parameters is usually provided by the transformer manufacturers, and the data for the zero sequence parameters could be obtained upon additional request. As a result of modeling, the vectors of the voltages and currents were obtained in all windings in the investigated modes with supply source phase failure. This makes it possible to analyze the admissibility of such modes and estimate the need for taking special measures of protection against them. It is shown that the voltages on the windings of the main transformer insignificantly depend on the connection mode of the regulating autotransformer, while the pattern of currents distribution in the windings of the main and regulating transformers to a large extent is determined by said connection mode of the autotransformer. The presence of perceptible zero-sequence current flowing through the grounded neutrals of the power supply source and load is noticed. This fact is connected with the release of insignificant magnetic flux from the magnetic circuit in the surrounding space.
\end{abstract}

Keywords: flexible connection of power systems, phase-shifting transformer, hexagon circuit diagram, regulating autotransformer, phase failure.

DOI:https://doi.org/10.52254/1857-0070.2021.3-51.02

UDC: 621.314 .2

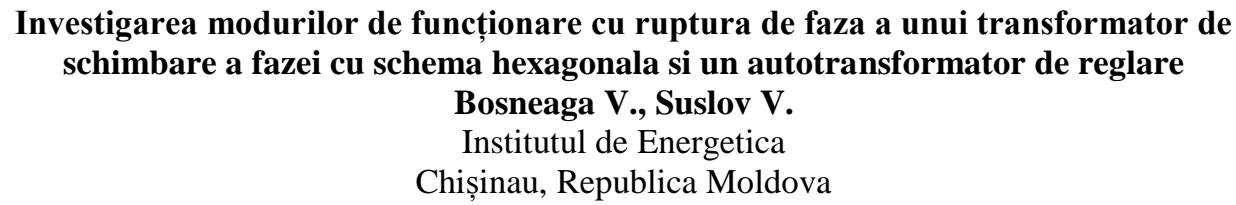

Rezumat. Dispozitivul propus are avantaje semnificative față de cele cunoscute și permite implementarea unei conectării flexibile la curent alternativ fără de a utiliza DC link. Prin urmare, scopul lucrării este studierea modurilor de funcționare cu ruptura de fază a sursei de alimentare și evaluarea admisibilității regimului dat pentru un transformator nou de reglare a decalajului de fază cu schemă hexagonală. Pentru a atinge scopul menționat a fost elaborat un model matematic al dispozitivului. Modelul include trei blocuri principale, elaborate de autori, care simulează două transformatoare cu trei coloane cu 6 înfășurări fiecare și un bloc de comutare a sarcinii. Transformatoarele se caracterizează prin două seturi diferite de parametri, bazate pe date experimentale pentru modurile de scurtcircuit și fără sarcină pentru secvențele directă și zero. Ca rezultat al calculelor efectuate au fost obținute valorile tensiunilor și curenților în toate înfăşurările în modurile de funcționare cu de fază. Aceasta a făcut posibilă analiza admisibilității acestor moduri cu concluzia, că nu este nevoie de a lua măsuri speciale pentru protecție împotriva lor. Este demonstrat, că tensiunile înfășurărilor transformatorului principal depind nesemnificativ de schema de conectare a autotransformatorului de reglare, în timp ce felul de distribuție a curenților în înfasșurările transformatoarelor principale și de reglare este determinat de schema de conectare a autotransformatorului. În aceste moduri, a fost dezvăluită prezența unui curent notabil de secvență zero, care curge prin neutrele împământate ale sursei de alimentare și ale sarcinii, care este asociat cu ieșirea unui mic flux magnetic în spațiul extern.

Cuvinte-cheie: ruptura de faza, transformator cu reglarea decalajului de fază, vectorii de curenți și tensiuni a înfășurărilor, curenții de secvență zero, distribuția fluxurilor magnetice relative.

(C) Bosneaga V., Suslov V., 2021 


\section{Исследование неполнофазных установившихся режимов работы фазоповоротного трансформатора по схеме шестиугольника с регулировочным автотрансформатором Бошняга В.А., Суслов В.М.}

Институт энергетики

Кишинев, Республика Молдова

Аннотация. Предлагаемое устройство обладает существенными преимуществами по сравнению с известными и позволяет реализовать гибкую связь энергосистем на переменном токе без использования звена постоянного тока. Важную роль при оценке реализуемости и других технических характеристик играет поведение устройства в неполнофазных режимах. Поэтому целью работы является исследование неполнофазных установившихся режимов работы и оценка их допустимости для нового фазоповоротного трансформатора по схеме многоугольника с дополнительным регулировочным автотрансформатором. Для решения этой задачи разработана математическая модель устройства. Модель включает три основных новых блока, два из которых моделируют основной и добавочный 6-ти обмоточные трехстержневые трансформаторы, имеющие разные наборы параметров. Параметры основаны на данных для режимов короткого замыкания и холостого хода для прямой и нулевой последовательности. Третий блок обеспечивает подключение нагрузки к нужным отводам автотрансформатора. В результате расчетов получены значения напряжений и токов во всех обмотках в неполнофазных режимах работы устройства, что позволило проанализировать допустимость таких режимов и сделать вывод о том, что нет необходимости принятия специальных мер по защите от них. Показано, что напряжения на обмотках основного трансформатора несущественно зависят от схемы подключения регулирующего автотрансформатора, а картина распределения токов в обмотках трансформаторов определяется схемой подключения автотрансформатора. Эта информация необходима для более точной оценки типовой мощности, определяющей расход активных материалов на изготовление устройства. Выявлено наличие заметного тока нулевой последовательности, протекающего через заземленные нейтрали источника питания и нагрузки, с чем связан выход небольшого магнитного потока во внешнее пространство. Значимость работы также, наряду с новыми результатами, заключается в том, что разработанная модель является универсальным удобным инструментом для исследования практически любых несимметричных режимов работы как данного устройства, так и других подобных.

Ключевые слова: неполнофазный режим, фазоповоротный трансформатор, вектора токов и напряжений обмоток, токи нулевой последовательности, распределение относительных магнитных потоков.

\section{ВВЕДЕНИЕ}

Известно, что фазоповоротные трансформаторы являются эффективным средством управления потоками мощности в сложнозамкнутых электрических сетях. В частности, они позволяют перераспределять загрузку параллельно работающих электропередач различного класса напряжения.

Кроме использования в стационарных режимах, при условии их обеспечения быстродействующими коммутирующими аппаратами, они могут приобретать свойства устройств типа FACTs (Flexible Alternating Current Transmission), что позволяет их также использовать для управления переходными процессами.

С учетом этой их новой роли и развития тенденции повышения управляемости электрических сетей (внедрения элементов smart grid) возрастает актуальность исследований различных новых технических решений, связанных с регулированием величины и фазы векторов напряжений в электрической сети.
Данная работа посвящена исследованию неполнофазных режимов работы новой схемы фазоповоротного трансформатора на базе многоугольника, дополненного регулировочным автотрансформатором, предложенной авторами в рамках заявки на получение патента республики Молдова. Предложенное устройство обладает определенными преимуществами перед известными схемами, заключающимися в том, что за счет существенного уменьшения количества необходимых регулировочных отводов упрощается конструкция основного трансформатора с соединением обмоток в многоугольник.

Кроме того, это влечет за собой соответствующее уменьшение необходимого количества коммутирующих элементов. Данное устройство, при условии оснащения быстродействующими полупроводниковыми ключами, может также выполнять функцию «гибкой» межсистемной связи на переменном токе, приобретая, таким образом, свойства устройств типа "FACTs.

В настоящее время обычно в качестве управляемых «гибких» межсистемных связей 
между несинхронно работающими энергосистемами переменного тока применяются вставки постоянного тока, которые получили достаточно широкое распространение в электрических сетях в Северной Америке и Европе [1-4].

Несмотря на присущие им некоторые недостатки, вставки постоянного тока являются единственным практически реализованным в широком масштабе решением, позволяющим независимо управлять потоками активной и реактивной мощности в линиях межсистемной связи, которое к тому же непрерывно совершенствуется и достигло высокой степени совершенства.

В данной работе рассматривается другое возможное техническое решение, базирующееся на достижениях твердотельной электроники $[5,6], \quad$ однако без промежуточного звена постоянного тока.

В связи бурным развитием в последние десятилетия технологий «FACTs» появилось большое количество различных управляемых с помощью полупроводниковых коммутаторов устройств. К ним можно отнести, например, устройства типа UPFC«Unified Power Flow Controller». Эти устройства обеспечивают регулирование параметров режима электрической сети, таких как величина и фаза напряжения в узле, потока активной и реактивной мощностей в сети $[7,8]$.

Однако, в доступной литературе не рассматривается возможность их адаптации и использования для обеспечения гибкой связи несинхронно работающих энергосистем. Причина в том, что при этом угол фазового сдвига между векторами напряжений систем должен изменяться в полном диапазоне 0$360^{\circ}$.

Ввиду изложенного, представляет интерес и становится реальным еще одно возможное направление реализации такой связи, основанное на применении устройств типа матричных циклоконвертеров [9-13], используемых в настоящее время для питания переменным током регулируемой частоты мощных электродвигателей.

Одним из недостатков, препятствующих их использованию для гибкой связи энергосистем, является большая дискретность регулирования угла, составляющая обычно $120^{\circ}$.
Это не позволяет удерживать направление и величину потока активной мощности по этой связи в необходимых пределах при заданных параметрах режима связываемых энергосистем.

В связи с вышесказанным, настоящая работа посвящена продолжению исследований новой схемы трансформаторного фазоповоротного устройства, предложенного авторами. Суть ее заключается в использовании трансформаторного фазоповоротного устройства, обеспечивающего круговое вращение фазы выходного напряжения относительно входного, которое фактически представляет собой преобразователь частоты ([14-16]).

В данной работе проведено подробное исследование неполнофазных режимов работы, которые могут возникать в процессе эксплуатации, с целью определения максимальных токов и напряжений обмоток устройства для оценки допустимой продолжительности таких режимов, а также необходимости применения мер защиты при их возникновении. Это позволит в дальнейшем, после более детального изучения других режимов работы, сделать вывод о перспективах практического использования предлагаемого технического решения.

\section{НЕПОЛНОФАЗНЫЕ РЕЖИМЫ УСТРОЙСТВА С ДОПОЛНИТЕЛЬНЫМ РЕГУЛИРОВОЧНЫМ АВТОТРАНСФОРМАТОРОМ}

Исследование неполнофазных режимов нового устройства (см. рис.1) проводилось с использованием пакета МАТЛАБСИМУЛИНК на базе модели, предложенной в более ранних работах авторов. Данная модель трехфазного трехстержневого трансформатора позволяет рассчитывать также и практически любые несимметричные режимы работы, в том числе и неполнофазные. В доступных опубликованных работах ([17-21]) отсутствует описание такого рода моделей, позволяющих исследовать несимметричные режимы работы трехфазных многообмоточных трансформаторов, реализованных для случая не менее 6 обмоток и имеющих нетрадиционную специальную схему соединения обмоток для получения фазового сдвига. Часть публикаций посвящены исследованию других режимов, в частности, 
в [17] рассмотрен только режим холостого хода, что не позволяет моделировать влияние нагрузки.

В [18] описан метод моделирования трехфазного трансформатора в фазных координатах на основе матрицы проводимостей, однако в работе могут моделироваться лишь простые классические схемы соединения обмоток.

В [19] также описан метод математического моделирования трехфазного силового трансформатора применительно к самому простому случаю 2-х обмоточного преобразовательного трансформатора, однако приведенный подход не позволяет рассматривать сколько-нибудь сложные схемы, характерные для преобразовательных или фазопреобразующих трансформаторов.

В [20] дано описание цифровой модели трехфазного трехстержневого трансформатора в среде МАТЛАБ применительно к случаю 2-х обмоток, что не позволяет решать задачи, связанные с расчетом схем, содержащих многообмоточные трансформаторы.

В публикации [21] автором заявлено создание модели многофазного многообмоточного трансформатора в МАТЛАБе, описана методика ее создания и подход, основанный на преобразовании Кларка. Однако де-факто приведена лишь методика построения модели только для случая трехфазного двухобмоточного трансформатора, что существенно ограничивает применение предложенного подхода.

Для данного исследования модель была несколько расширена для обеспечения возможности моделирования двух отдельных трансформаторов и добавлена возможность моделирования до 6-ти обмоток. Один из трансформаторов-это основной фазоповоротный трансформатор с обмотками, соединенными в шестиугольник. Для определенности, выберем величину шага регулирования угла в $5^{\circ}$, тогда, для его обеспечения, регулировочный автотрансформатор, перекрывающий диапазон в $30^{\circ}$, должен иметь обмотку, состоящую также из 6 частей с отводами, т.е. его также можно моделировать в данном случае 6-ти обмоточным трансформатором.

При необходимости уменьшить ступень регулирования угла надо будет увеличить число промежуточных отводов регулировочного автотрансформатора. Либо можно использовать известный прием, когда нужная величина ступени обеспечивается комбинацией относительно небольшого числа обмоток с разными числами витков, описанный, например в [22]. Параметры трансформаторов взяты такими же, как и в предыдущей работе авторов.

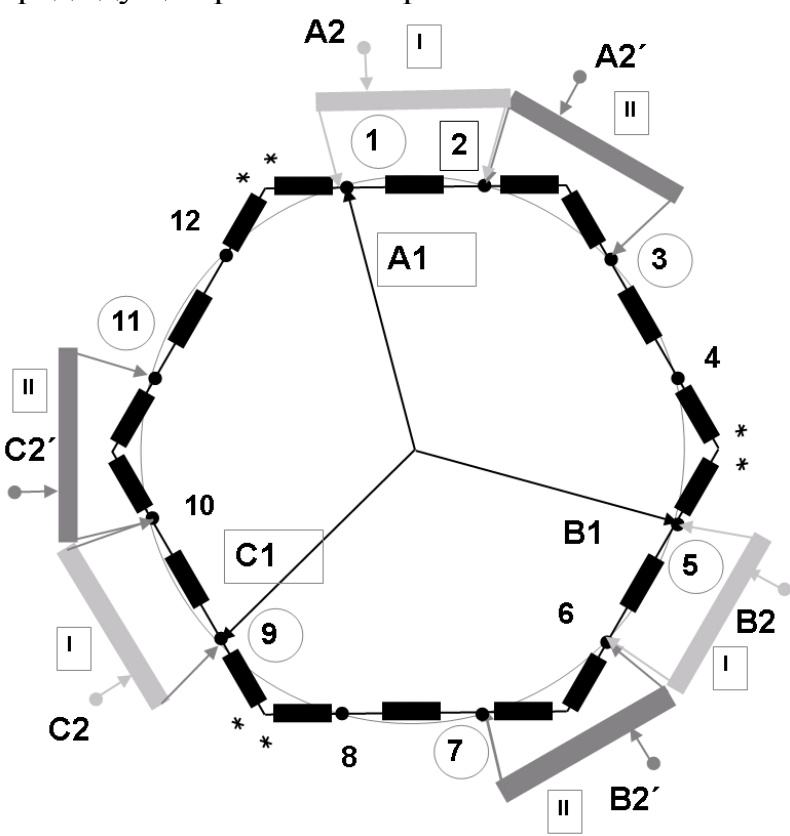

Рис.1. Условная схема предложенного устройства для гибкой связи энергосистем.

Fig. 1. Conventional diagram of the proposed device for flexible connection of power systems.

На рисунке 1 приведена условная схема, поясняющая идею работы устройства, причем трансформаторные обмотки нарисованы в виде зачерненных прямоугольников так, чтобы их продольная ось совпадала с направлениями векторов, наводимых в них э.Д.с.

Начала обмоток обозначены звездочками. Оси обмоток разных фаз изображены для наглядности с геометрическим сдвигом на $120^{\circ}$. Обмотки фаз регулировочного автотрансформатора, подключаемые к различным смежным отводам основного трансформатора (которые пронумерованы цифрами от 1 до 12) показаны условно двумя более светлыми градациями штриховки, каждая из которых относится к одному из его возможных положений.

Одно из возможных положений обозначено буквами А2, В2, С2, а второе А2',В2',С2'. В процессе регулирования угла регулировочный трансформатор может подключаться на все остальные следующие по кругу смежные отводы основного 
трансформатора, обеспечивая изменение угла скачком на величину $30^{\circ}$, а плавное регулирование осуществляется с помощью регулировочных отводов автотрансформатора.

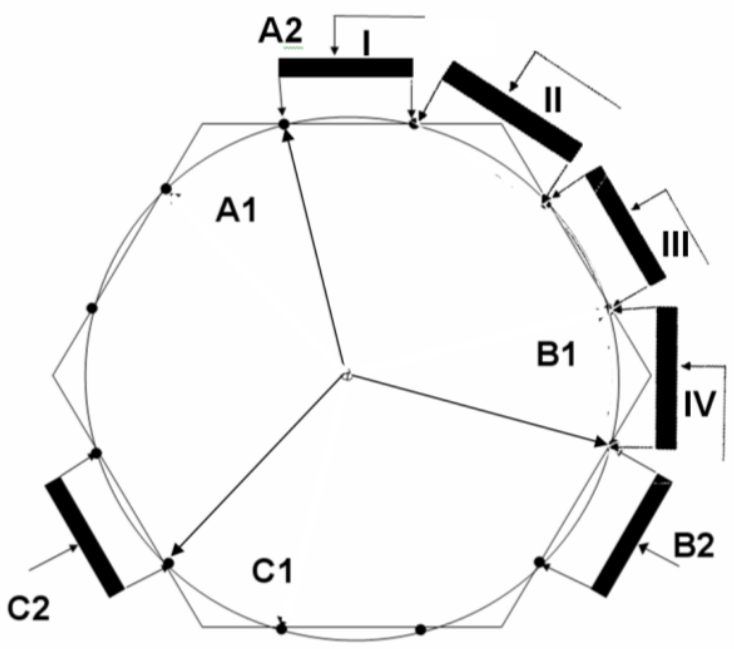

Рис. 2. Схема, поясняющая различные возможные положения регулирующего автотрансформатора.

Fig. 2. Diagram explaining the various possible positions of the regulating autotransformer.

На рис.2 показаны различные возможные положения регулирующего автотрансформатора, которые дают отличающиеся режимы работы устройства. Как видно, их всего 4, для каждого положения характерен свой режим работы, отличный от остальных в силу различного подключения автотрансформатора и питающего напряжения к отводам многоугольника.

Рассмотрим по очереди все 4 возможные положения регулирующего автотрансформатора при обрыве фазы А питающей системы напряжений. Расчетная схема для режима I показана на рис. 3, аналогичным образом, только с подключением регулирующего автотрансформатора к следующей по кругу паре отводов будут выглядеть остальные 3 схемы подключения автотрансформатора.

На рис.3 буквами А, В, С условно обозначены обмотки соответствующих фаз трансформатора, их номера проставлены рядом с ними в кружках, так что отдельную обмотку можно идентифицировать, например, как A1. Каждая фаза состоит из двух групп по 3 обмотки, соединенных последовательно и согласно и составляющих противоположные стороны шестиугольника.
Система питающих напряжений приложена к началам обмоток А2, В2, C2. Фазы регулирующего автотрансформатора

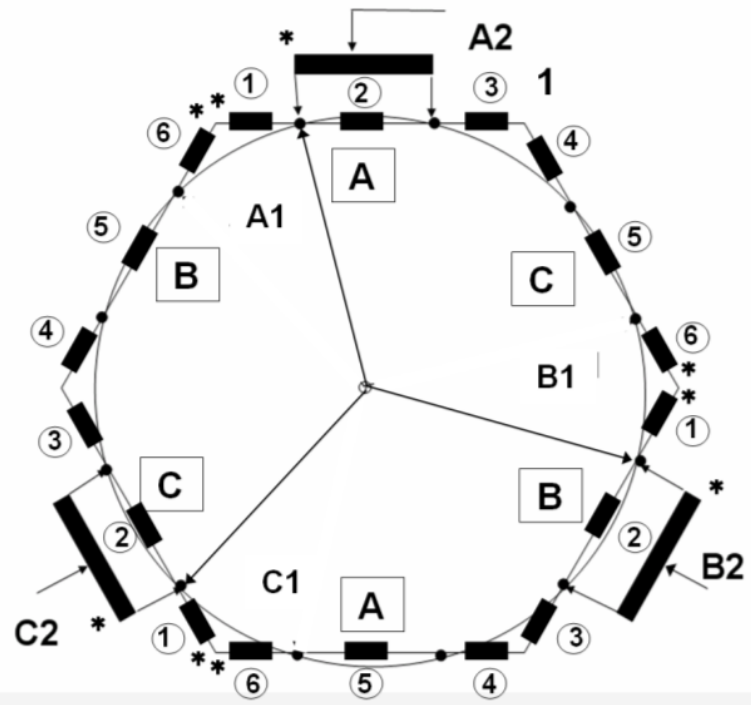

Рис.3. Расчетная схема для первого варианта подключения регулирующего автотрансформатора (режим I).

Fig.3. Calculation scheme for the regulating autotransformer connection (mode I).

также обозначены теми же буквами А, В, С и подключены, как видно из рис.3, к соответствующим симметрично расположенным обмоткам шестиугольника. Например, концы обмотки фазы А автотрансформатора подключены в данном случае к началам обмоток А2 и А3.

Место подключения нагрузки отображается условно стрелками, подходящими к регулировочной обмотке автотрансформатора. В данной работе будем считать ее подключенной к средним отводам автотрансформатора, одинаково для всех трех фаз.

Отметим, что модель позволяет рассчитать режим и для случая неодинакового подключения нагрузок по фазам, однако эти режимы планируется рассмотреть в другой работе.

Модель состоит из нескольких блоков, разработанных авторами. Используются также стандартные блоки СИМУЛИНКа для питающей трехфазной энергосистемы с заданными параметрами в режиме с заземленной нейтралью и блок нагрузки с заданным сопротивлением.

Первый из новых блоков моделирует основной фазоповоротный трансформатор с 6-ю обмотками в каждой фазе, соединенными в шестиугольник. Второй же моделирует 
трехстержневой автотрансформатор с регулировочной обмоткой, состоящей из последовательно включенных 6-ти обмоток с отводами от каждой обмотки (всего 7 отводов).

В модели предусмотрена настройка независимого переключения этих отводов к каждой фазе нагрузки. Оба эти блока инициируются заданными первичными параметрами при запуске модели в работу.

Bce соединения между отдельными обмотками в обоих блоках вынесены за их пределы и выполнены авторами вручную при сборке схемы соединения обмоток в модели. Доступны для подключения начала и концы всех обмоток всех трех фаз обоих трансформаторов, которые можно соединить в любом нужном сочетании в соответствии с исследуемой схемой соединения обмоток.

Кроме того, заданы номинальные параметры обоих трансформаторов, такие как номинальная мощность и номинальное напряжение для основного трансформатора (63 МВА, 110 кВ), потери в режиме короткого замыкания и ток холостого хода, a также парные напряжения короткого замыкания пар обмоток для прямой и нулевой последовательности. Эти данные менее доступны, обычно изготовители трансформаторов не публикуют их в полном объеме, однако они могут быть получены по дополнительному запросу.

Более подробно с методикой задания исходных данных можно ознакомиться в работе [18]. Нагрузка в данном случае моделируется одинаковыми активными сопротивлениями, однако могут также быть заданы и комплексные сопротивления нагрузки, причем можно их задавать различными для каждой из фаз при моделировании реактивной мощности нагрузки и ее несимметрии по фазам. Такие режимы планируется рассмотреть в отдельной работе.

Отметим, что данная модель достаточно универсальна, так как позволяет исследовать и любую другую схему соединения обмоток, достаточно пересоединить обмотки в нужном порядке.

Рассмотрим один из возможных режимов (режим I, см. рис.2), когда оба вывода фазы регулировочного автотрансформатора подключены к обмоткам только одной фазы, а именно к той, к которой приложена система питающих напряжений.
Результаты расчетов будем представлять в виде векторных диаграмм (ВД) напряжений и токов, которые, в отличие от табличной формы представления результатов, в компактной форме дают полное и наглядное представление об особенностях изучаемых режимов.

Сначала рассмотрим режим холостого хода, при котором нагрузка отсутствует. Полученные для этого режима результаты расчета напряжений обмоток многоугольника для случая обрыва фазы А представлены в виде ВД на рисунке 4.

Вектора напряжений обмоток разных фаз изображены сплошными линиями. Обозначены вектора напряжений A1, A2, A3 и т.д. для фазы А и аналогично для фаз В и С. Приложенным напряжениям соответствуют вектора В и С. Масштаб напряжения также приведен на данном рисунке.

При наличии только двух фаз питания, к двум точкам многоугольника обмоток приложено однофазное напряжение, равное линейному напряжению питающей системы между фазами В и С. Поэтому шестиугольник напряжений (характерный, например, для симметричного режима) вырождается в отрезок практически прямой линии, однако для наглядности накладывающиеся друг на друга вектора напряжений обмоток изображены на рис.4 условно на некотором расстоянии.

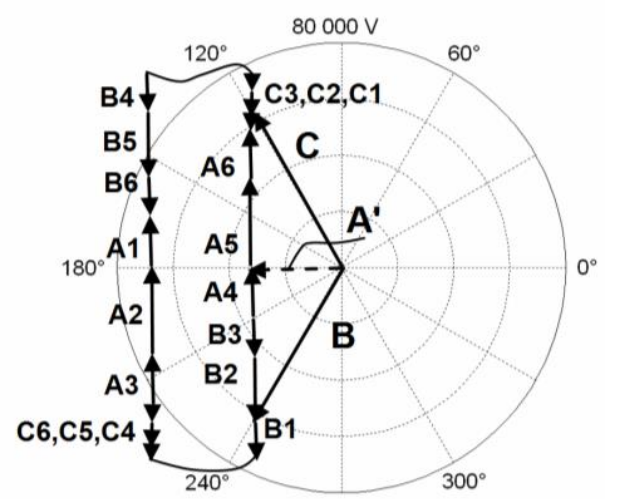

Рис. 4. ВД напряжений обмоток многоугольника для случая обрыва фазы А без нагрузки.

Fig. 4. Vectorial diagram (VD) of polygon windings voltages at open phase $A$ at no load.

При этом концы векторов напряжений, которые разнесены, соединены условно кривыми сплошными линиями. Вектор $\mathrm{A}^{\prime}$ обозначает величину напряжения, которое 
будет в точке соединения обмоток А1 и А2, где ранее была подключена фаза А питания.

Отметим, что, как показали расчеты, величины напряжений обмоток в неполнофазном режиме холостого хода меньше или близки к их номинальным значениям, поэтому перенапряжения на обмотках отсутствуют.

На следующем рис. 5 показаны относительные магнитные потоки в стержнях магнитопровода. Как видно из рис.5, все потоки, расположены вдоль одной прямой (что согласуется с ВД напряжений обмоток на рис.4), при этом потоки стержней В и С направлены противоположно потоку фазы А. Сумма потоков всех трех стержней, как показал расчет, равна нулю. Это означает, что в данном режиме поток замыкается внутри магнитопровода, не выходя в окружающее пространство.

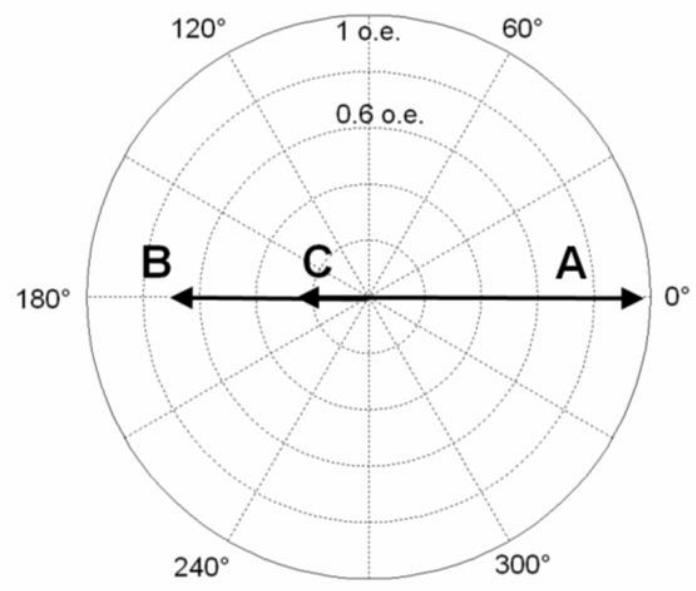

Рис. 5. Относительные магнитные потоки в стержнях магнитопровода.

Fig. 5. Relative magnetic fluxes in the legs of the magnetic circuit.

Полученные результаты для режима холостого хода можно распространить и на остальные схемы подключения регулирующего автотранс-форматора, так как при отсутствии нагрузки результаты будут аналогичными.

Рассмотрим далее неполнофазные режимы при наличии нагрузки. Полученные результаты расчета напряжений обмоток многоугольника для случая обрыва фазы А в схеме I при наличии нагрузки представлены на рисунке 6. Здесь буквами А, В, С обозначена принадлежность векторов напряжений обмоткам соответствующих фаз основного трансформатора. Цифры, проставленные возле векторов, обозначают

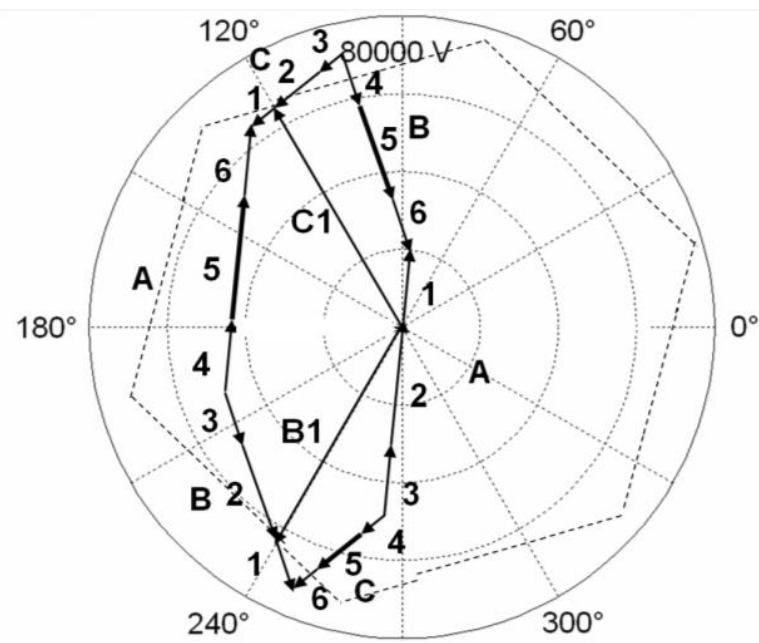

Рис. 6. ВД напряжений обмоток основного трансформатора при обрыве фазы А при нагрузке.

Fig. 6. Vectorial diagram (VD) of polygon windings voltages in case of phase A break at load.

номера этих обмоток. Тонким пунктиром отображен шестиугольник напряжений обмоток для нормального режима нагрузки. Показаны также вектора приложенных фаз $\mathrm{A} 1, \mathrm{~B} 1, \mathrm{C} 1$. Вектор напряжения A1' в точке шестиугольника, где ранее была приложена оборванная фаза A1 не показан, так как слишком мал в данном режиме.

Из этого рисунка видно, что вектора напряжений обмоток основного трансформатора образуют существенно искаженный и значительно сплюснутый по горизонтали по сравнению с нормальным симметричным режимом шестиугольник.

Величины напряжений одноименных обмоток разных фаз в этом режиме отличаются друг от друга, а напряжения противолежащих обмоток шестиугольника примерно равны друг другу.

Косвенным подтверждением правильности полученных результатов является то, что построенный на основе рассчитанных векторов напряжений обмоток шестиугольник получается замкнутым в соответствии со схемой соединения обмоток. Также, как и в предыдущем случае при холостом ходе, перенапряжения на обмотках отсутствуют.

Рассмотрим далее токи обмоток устройства. Сначала в качестве базы для сравнения приведем рассчитанные токи симметричного нормального режима 
нагрузки для основного трансформатора в данном случае. Они показаны на рис. 7. Рассматривая ВД рис.7 можно убедиться в том, что все рассчитанные токи соответствуют схемным условиям. Например, видно, что вектора токов А4, А5, А6 совпадают. В то же время, токи частей обмоток, принадлежащих другим фазам,

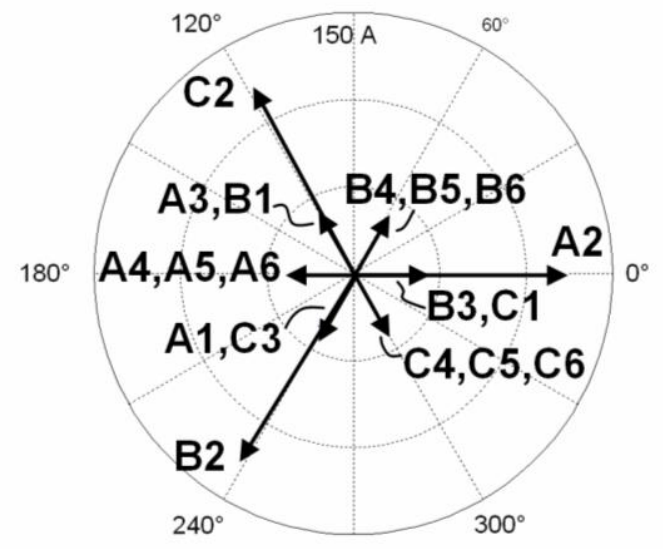

Рис.7.Токи обмоток основного трансформатора в симметричном режиме нагрузки в схеме I.

Fig. 7. The currents of the main transformer windings in symmetrical load mode in scheme $I$.

прилегающие к упомянутым обмоткам и также соединенные последовательно с ними (B3, C1), находятся в противофазе, в соответствии со схемой соединения обмоток и разметкой начал обмоток. Отметим, что наибольшие токи возникают в обмотках А2, B2, C2, параллельно которым подключен регулирующий автотрансформатор. Далее рассмотрим токи при обрыве питания фазы A.

На рис.8 изображены вектора токов в обмотках основного трансформатора в рассматриваемом неполнофазном режиме.

Как видно из данного рисунка, все токи также соответствуют схемным условиям рис. 3. Аналогично предыдущему, токи обмоток A4, A5, А6 одинаковы. Токи обмоток В3, C1 равны им по модулю, но противоположны по фазе, так как текут в другом направлении относительно начал соответствующих обмоток. Аналогичная закономерность наблюдается и относительно совокупностей токов обмоток B4,B5,B6 и A1,C3, а также $\mathrm{C} 4, \mathrm{C} 5, \mathrm{C} 6$ и А3,B1.

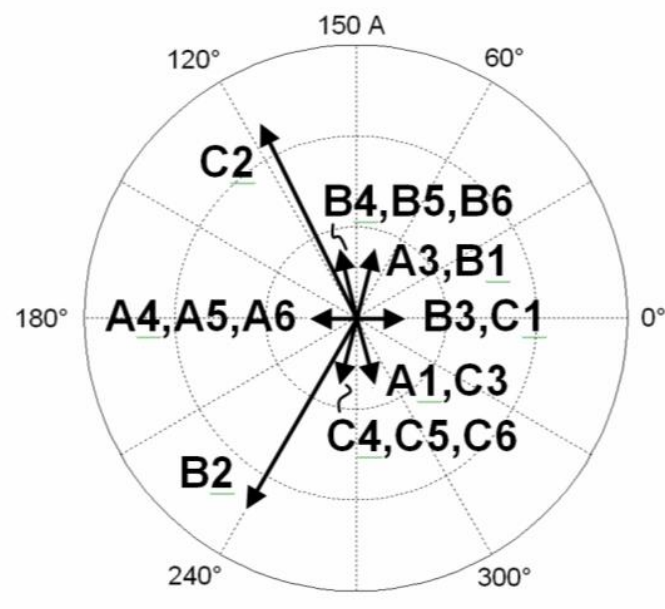

Рис.8. ВД токов в обмотках основного трансформатора в неполнофазном режиме.

Fig. 8. VD of currents in the main transformer windings at open-phase mode.

Как следует из сравнения рис. 7 и 8, токи всех обмоток по модулю остались практически теми же, изменились лишь незначительно их фазовые углы. Исключением является ток в обмотке А2, который значительно уменьшился при обрыве питания фазы А, поэтому на ВД рис. 8 он не показан. В данном режиме в рассматриваемой схеме превалирует загрузка по току обмоток под номером 2 для фаз В и С по сравнению с другими обмотками.

Кроме того, следует также отметить, что для рассчитанных токов (и в дальнейшем для всех случаев), как показал анализ, соблюдается 1-й закон Кирхгофа для токов в узлах, где регулирующий автотрансформатор подключен к основному трансформатору.

Рассмотрим далее токи в обмотках регулирующего автотрансформатора.

Отметим, что на рис. 9 в качестве базы для сравнения приведены данные токи при симметричном режиме нагрузки.

Результаты моделирования показали, что по величине данные токи приблизительно одинаковы для всех четырех рассмотренных схем подключения. Разница состоит лишь в фазовых углах, что объясняется различными схемами подключения регулирующего автотрансформатора. 


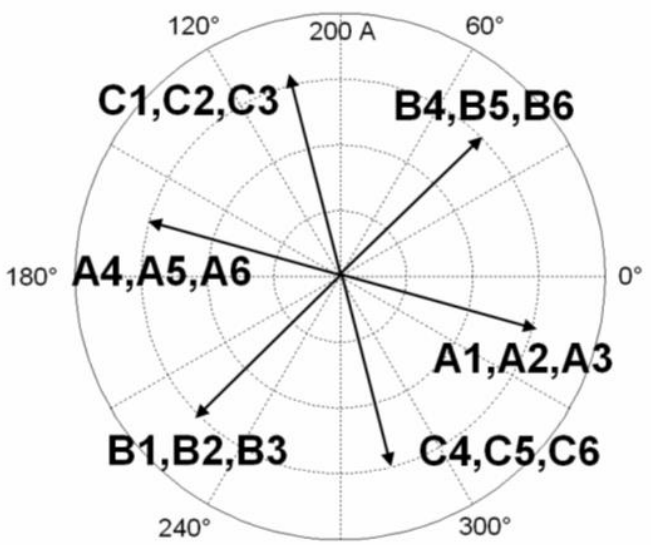

Рис.9. ВД токов в обмотках регулирующего автотрансформатора при симметричном режиме нагрузки.

Fig. 9. VD of currents in the regulating autotransformer windings at symmetrical load mode.

Далее на рис.10 показана ВД токов для неполнофазного режима в обмотках регулирующего автотрансформатора. Нагрузка при этом, как и везде ранее, подключена к серединам регулировочных обмоток. Аналогично предыдущему, соблюдаются закономерности для токов в соответствии со схемой соединения.

Видно также, что в данном режиме наименьшие токи протекают по обмоткам (A1-А6) регулирующего автотрансформатора, подключен-

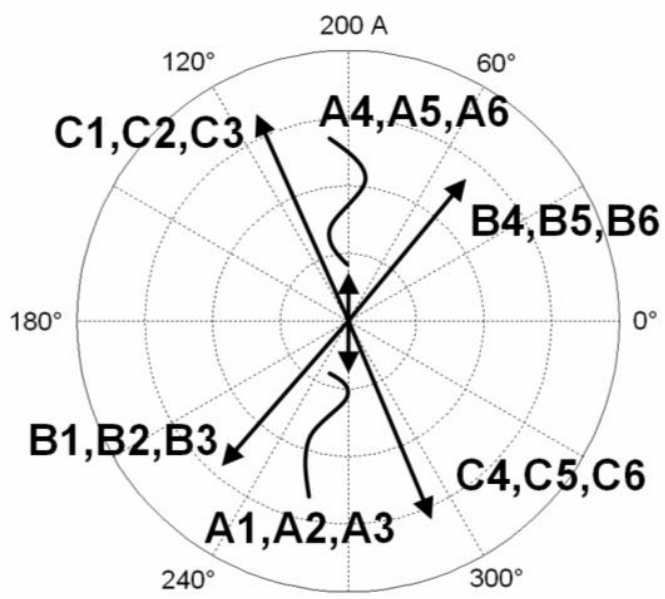

Рис.10. ВД токов регулирующего автотрансформатора в неполнофазном режиме. Fig. 10. VD of currents of the regulating autotransformer windings in open-phase mode.

ного к фазе А основного трансформатора, где отключено питание. Таким образом, как следует из сравнения рисунков 9 и 10 , токи «здоровых» фаз не претерпели кардинальных изменений.

На следующем рис. 11 приведена ВД токов нагрузки. Как видно из данного рисунка, при обрыве питания фазы А, ток нагрузки в этой фазе все-таки есть за счет того, что со схемы многоугольника через соответствующую фазу регулирующего автотрансформатора на нагрузку поступает некоторое напряжение.

Но величина тока значительно меньше токов двух «здоровых» фаз. Кроме того, как видно из данного рисунка, сумма токов фаз

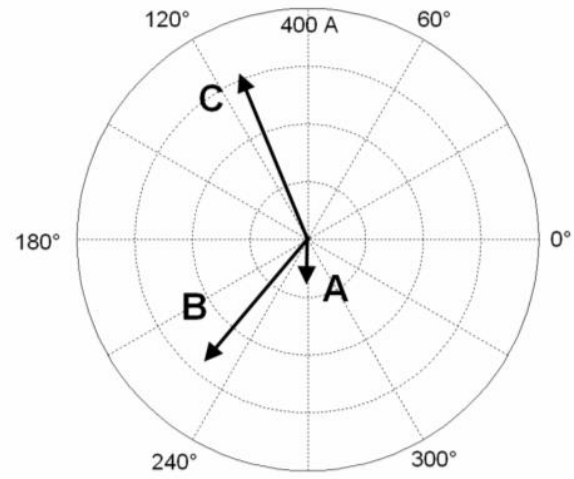

Рис. 11. ВД токов нагрузки.

Fig. 11. VD of load currents.

нагрузки не равна нулю, что означает, что через заземленные нейтрали нагрузки и источника питания циркулирует заметный ток, сравнимый с токами нагрузки «здоровых» фаз.

Таким образом, данный режим характеризуется наличием значительного тока нулевой последовательности. Это позволяет предположить, что и магнитные потоки в стержнях трансформаторов могут иметь некоторую составляющую нулевой последовательности.

На рис. 12 показана ВД относительных магнитных потоков в стержнях основного трансформатора. Видно, что относительные потоки стержней несимметричны, однако их сумма, как показал расчет, почти равна нулю. Имеется лишь небольшой поток, выходящий за пределы магнитопровода величиной в $6 \%$ от полного потока. T.e., в данном несимметричном нагрузочном режиме появляется небольшой магнитный поток нулевой последовательности. Рассмотрим далее следующий возможный неполнофазный режим, возникающий в случае подключенного положения II 
регулирующего автотрансформатора в соответствии с схемой рис. 2.

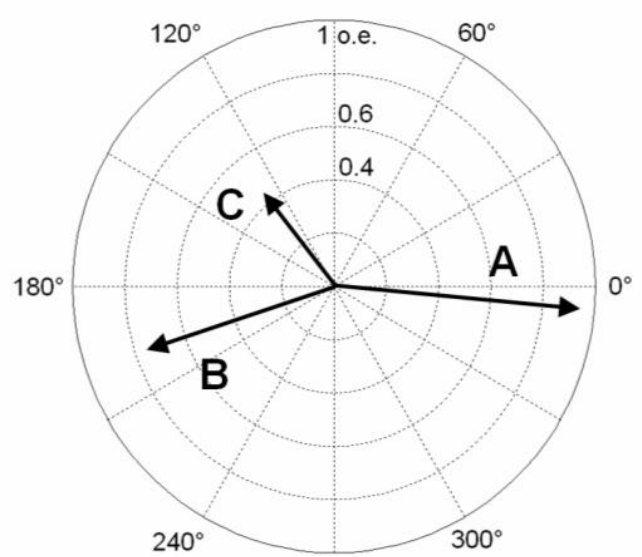

Рис. 12. ВД относительных магнитных потоков в стержнях основного трансформатора.

Fig. 12. VD of relative magnetic fluxes in the legs of the main transformer.

Его моделирование показало, что ВД напряжений обмоток основного трансформатора имеет вид, аналогичный предыдущему случаю.

В то же время распределение токов по обмоткам основного трансформатора кардинально отличается от предыдущего случая, что иллюстрируется ВД на рис. 13.

Как и в предыдущем случае, закономерности распределения токов отвечают схемным условиям. Наибольший по модулю ток наблюдается в обмотке С2, который, однако, лишь незначительно превышает ток нормального режима. Несколько меньший нормального ток течет по обмотке В2, и он меньше тока С2. Как следует из схемы рис. 3 , при обрыве питания фазы А, токи обмоток A1, А2 основного трансформатора равны по модулю токам В5, B6 и находятся по отношению к ним в противофазе, в соответствии с ВД рис.13.

То же самое соблюдается для токов С5, С6 и В1 и для токов обмоток разных фаз, находящихся между точками подключения регулирующего автотрансформатора и соединенных последовательно. Равны по модулю и противоположны по фазе пары токов А 3 и С4, В3 и А4, В4 и С3.

Таким образом, приведенные на ВД рис. 13 токи отвечают схемным условиям данного режима, что является дополнительным подтверждением правильности расчета.

Рассмотрим далее токи в обмотках регулирующего автотрансформатора. На рис.14 показана ВД токов в обмотках регулирующего автотрансформатора.

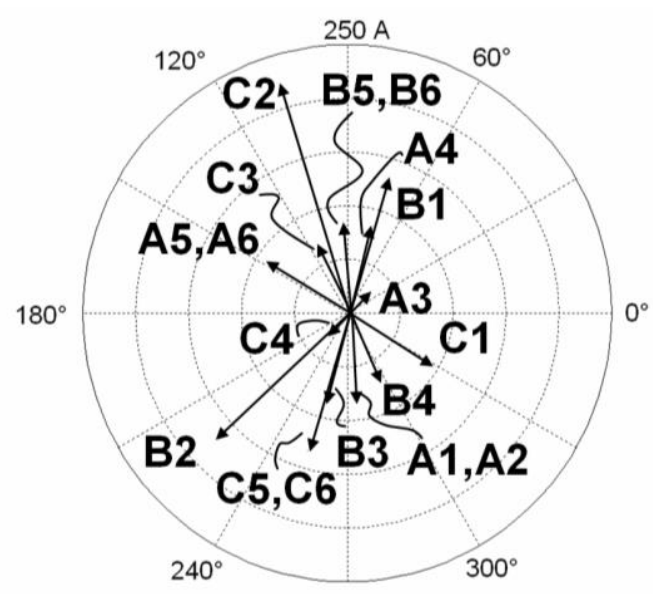

Рис.13. ВД токов обмоток основного трансформатора для подключения регулирующего трансформатора по схеме II.

Fig. 13. VD of main transformer currents at autotransformer connection by scheme II.

Из данной диаграммы видно, что для токов соблюдаются те же закономерности, которые

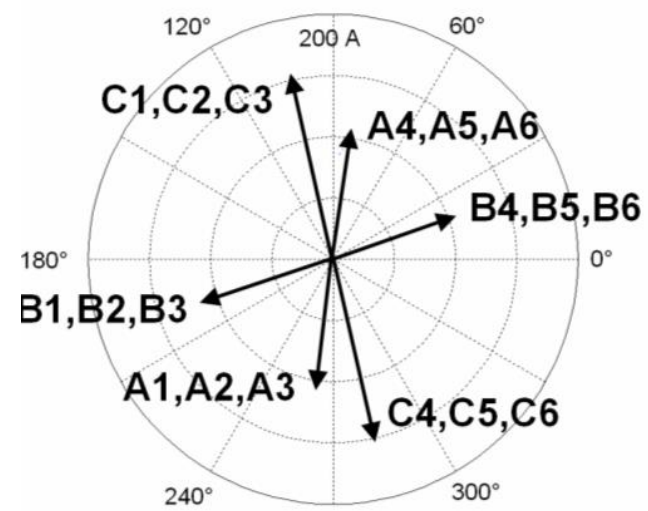

Рис.14. ВД токов в обмотках

регулирующего автотрансформатора.

Fig. 14. VD of regulating autotransformer currents.

были отмечены ранее.

Как можно заметить из сравнения с рис. 10, при данном подключении регулировочного автотрансформатора токи по его обмоткам распределены более равномерно и нет такой ярко выраженной разницы в величинах токов обмоток разных фаз.

И в этом случае, как и в ранее рассмотренном, между трехфазным источником и нагрузкой циркулирует некоторый ток нулевой последовательности. 
Рассмотрим далее следующий возможный неполнофазный режим, возникающий в случае подключения регулирующего автотрансформатора в положение III в соответствии со схемой рис. 2.

Моделирование показало, что ВД напряжений обмоток основного трансформатора имеет вид, аналогичный предыдущему случаю.

В то же время распределение токов по обмоткам основного трансформатора существенно отличается от предыдущего случая, что иллюстрируется ВД на рис. 15.

Как и во всех предыдущих случаях, закономерности распределения токов отвечают схемным условиям и согласуются с 1-м законом Кирхгофа. Наибольший по модулю ток наблюдается в обмотках В1,C6,

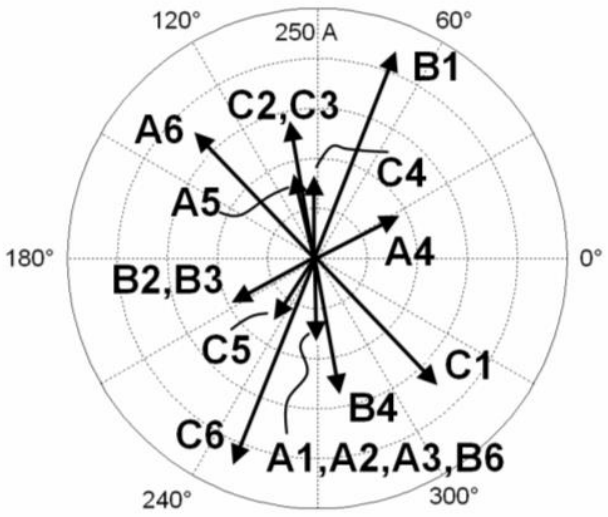

Рис.15. ВД токов основного трансформатора при обрыве фазы А питания.

Fig. 15. VD of the main transformer currents in the case of phase A break.

который, однако, лишь незначительно превышает ток нормального режима. Несколько меньшими по сравнению с нормальным являются токи, текущие по обмоткам А6,C1.

Как следует из схемы рис. 3, в данном режиме токи обмоток A1,A2,A3,B6 и C4 основного трансформатора равны по модулю (ток C4 находится в противофазе), что и подтверждается ВД рис. 15 .

Аналогичная закономерность соблюдается для токов В2,B3,A4 и С2,С3,B4. Отметим, что ток В5 намного меньше остальных токов и поэтому на ВД рис. 15 не показан.

Рассмотрим далее токи в обмотках регулирующего автотрансформатора. На рис.16 показана ВД токов в обмотках

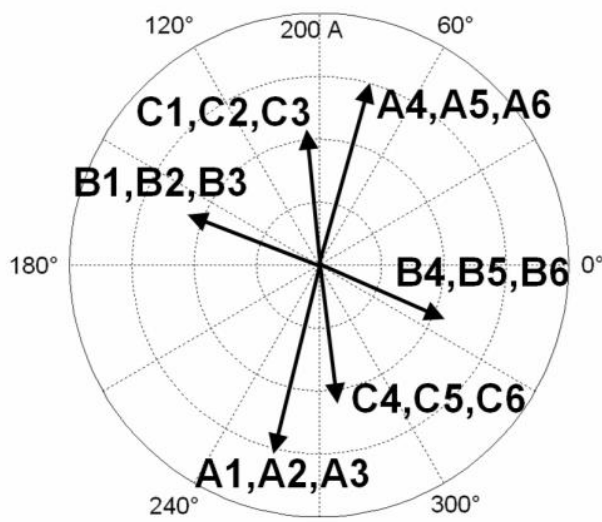

Рис.16. ВД токов в обмотках регулирующего автотрансформатора при обрыве фазы А.

Fig.16. VD of the regulating transformer currents for circuit IV at phase A break.

регулирующего автотрансформатора. На данном рисунке одинаковые токи в последовательно включенных частях регулировочной обмотки обозначены одним вектором. По величине все токи меньше токов нормального симметричного режима нагрузки.

Рассмотрим далее последний из возможных режимов, возникающий в случае подключения регулирующего автотрансформатора в положение IV в соответствии с схемой рис. 2.

Моделирование показало, что ВД напряжений обмоток основного трансформатора как в симметричном режиме нормальной нагрузки, так и при обрыве фазы питания А имеет вид, аналогичный предыдущему. То есть, напряжения обмоток шестиугольника практически не зависят от схемы подключения регулирующего автотрансформатора.

Анализ токов в обмотках основного трансформатора показал, как и ожидалось, что в токи в нормальном режиме нагрузки имеют величины, аналогичные тому, какие наблюдаются в схеме I. Это, видимо, объясняется тем, что как в схеме I, так и в схеме IV, точки подключения регулирующего автотрансформатора расположены ближе к питающим напряжениям.

Картина токов в обмотках основного трансформатора для рассматриваемого случая приведена на рис. 17. Отметим, что токи в обмотках A1 и В6 на ВД рис. 17 не показаны, поскольку они значительно меньше остальных 


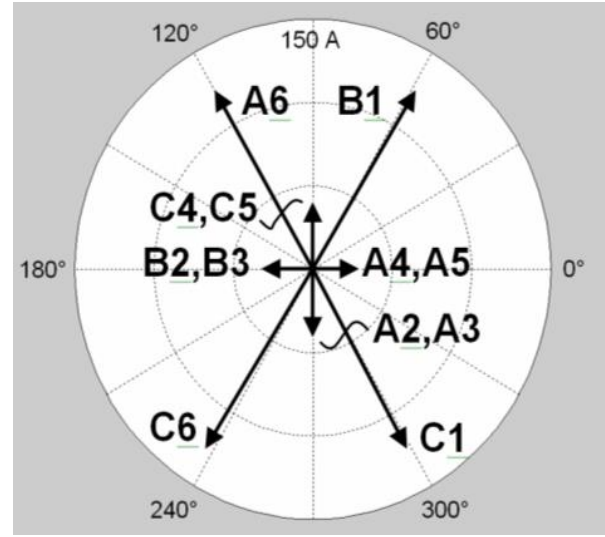

Рис. 17. ВД токов в обмотках основного трансформатора для схемы IV.

Fig. 17. VD of the main transformer currents for case IV at phase A break.

токов. Можно заметить, что наименьшее значение имеют токи в обмотках, примыкающих к точке подключения оборванной фазы A(A2-A5, B2,B3,C4,C5), а наибольшее значение имеют токи в обмотках, к которым подключены две фазы регулирующего автотрансформатора.

Также здесь, как и везде ранее, наблюдается полное соответствие рассчитанных значений токов схемным условиям, в чем можно убедиться при детальном рассмотрении ВД рис. 17 .

На рис.18 показана ВД токов в обмотках регулирующего автотрансформатора в неполнофазном режиме. Видно, что наименьшие токи имеют место в обмотках той фазы автотрансформатора, которая подключена одним из концов к точке подключения оборванной фазы А.

Отметим, что и в этом случае между трехфазным источником и нагрузкой циркулирует существенный ток нулевой последовательности.

Во всех вариантах подключения регулирующего трансформатора магнитные потоки практически не выходят за пределы сердечников как основного трансформатора, так и дополнительного автотрансформатора.

Дополнительный анализ величин токов в обмотках в симметричных режимах показал, что наиболее нагруженными обмотками в процессе вращения фазы оказываются 1,2 и 6 обмотки. Остальные обмотки могут быть выполнены на меньшие номинальные токи.

При обрывах фазы наиболее нагруженными оказываются эти же обмотки, однако токи в них лишь незначительно превышают токи симметричного нормального режима.

Вследствие этого принятие специальных мер для ограничения продолжительности существования неполно-фазного режима с точки зрения теплового эффекта не требуется.

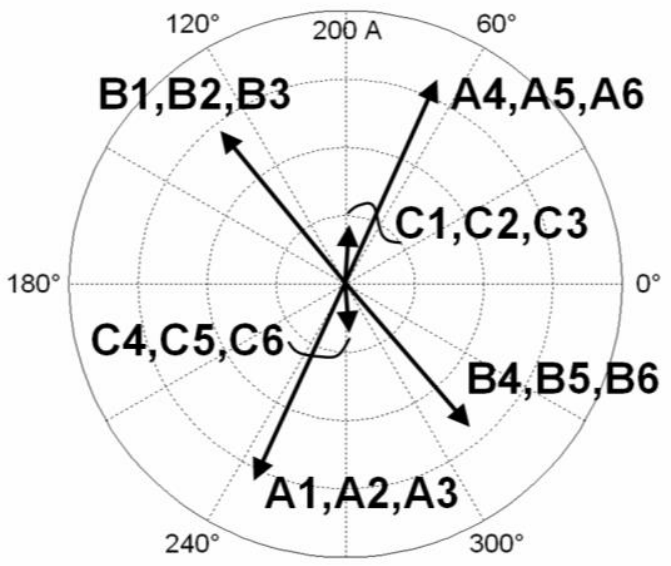

Рис.18. ВД токов в обмотках регулирующего автотрансформатора в неполнофазном режиме в схеме IV.

Fig.18. VD of the regulating transformer currents for circuit IV at phase break.

При обрывах фазы наиболее нагруженными оказываются эти же обмотки, однако токи в них лишь незначительно превышают токи симметричного нормального режима.

Вследствие этого принятие специальных мер для ограничения продолжительности существования неполно-фазного режима с точки зрения теплового эффекта не требуется.

Таким образом, предложенная модель устройства позволяет рассчитывать токи и напряжения обмоток нового двухтрансформаторного устройства для связи энергосистем на базе схемы шестиугольника в несимметричных режимах, при любых вариантах подключения регулирующего автотрансформатора и состояниях переключающих ключей.

\section{ВЫВОДЫ}

1. Разработана математическая модель нового трансформаторного трехфазного устройства для связи энергосистем на переменном токе, содержащего два 
трансформатора-основной фазоповоротный и дополнительный автотрансформатор, регулировочный позволяющая рассчитывать и исследовать как симметричные, так и практически любые несимметричные режимы рассмотренной схемы. Кроме того, она позволяет анализировать также и другие схемные решения, имеющие аналогичный набор трансформаторов, и является, таким образом, удобным инструментом для исследования трансформаторных устройств.

2. Получены значения токов и напряжений обмоток устройства во всех возможных положениях регулирующего автотрансформатора при подключении нагрузки к средним отводам его обмотки. Построены наглядные ВД, поясняющие особенности рассмотренных режимов работы предложенного устройства. Проведенные расчеты показали, что величины возникающих в неполнофазных режимах токов и напряжений обмоток находятся в допустимых пределах и не требуется принятия специальных мер защиты от таких режимов. Показано, что в неполнофазном режиме при заземленных нейтралях источника питания и нагрузки возможно протекание значительных токов нулевой последовательности через устройство, связанных с выходом небольшого магнитного потока во внешнее пространство.

3. Разработанная модель и полученные с ее помощью результаты позволяют также провести дальнейшие исследования характеристик устройства и в других несимметричных режимах с целью изучения перспектив создания нового устройства гибкой межсистемной связи между асинхронно работающими энергосистемами переменного тока без звена постоянного тока.

\section{Библиография (References)}

[1] Pan J., Nuqui R., Srivastava K., Jonsson T., Holmberg P., Hafner Ying-Jiang. AC Grid with Embedded VSC-HVDC for Secure and Efficient Power Delivery. IEEE Energy2030, Atlanta, GA USA, 17-18 November, 2008.

[2] Yu J., Karady G., Lei G., "Applications of Embedded HVDC in Power System Transmission", IEEE Power Engineering and Automation Conference (PEAM), September 2012.

[3] Jacobson B., Karlsson P., Asplund G., Harnefors L., Jonsson T., "VSCHVDC, Transmission with Cascaded Two-Level Converters", CIGRE Conference 2010, Paris, France.
[4] Marz M., Copp K., Manty A, Dickmander D., Danielsson J., Johansson F., Holmberg P., Bjorklund P., Duchen H., Lundberg P., Irwin G., Sankar S., Mackinac. "HVDC Converter-Automatic Runback Utilizing Locally Measured Quantities", CIGRE Conference, 2014, Toronto, Canada.

[5] IGBT Application Note, R07AN0001EJ0410, Rev.4.10, Jul 13, 2018, (C) 2018 Renesas Electronics Corporation.

URL

https://www.renesas.com/eu/en/doc/products/igbt/apn /r07an0001ej0410_igbt.pdf.

[6] Toshiba Electronic Devices \& Storage Corporation. IGBTs (Insulated Gate Bipolar Transistor) Application Note 2018-09-01 (C) 2018, 40pp.

URL https://toshiba.semicon-storage.com/apen/semiconductor/product/bipolar-transistors-

igbt.html. (accsessed 18.08.2021)

[7] Ketan G. Damor, Dipesh M. Patel, Vinesh Agrawal, Hirenkumar G. Patel. Comparison of Different Fact Devices. IJSTE-International Journal of Science Technology \& Engineering. Vol. 1, Issue 1, July 2014.

[8] Gyugyi L., Schauder C.D., Williams S.L., etc. The Unified Power Flow controller: a new approach to power transmission control. IEEE Transactions on Power Delivery, Vol.10, No.2, April 1995. pp.10851097.

[9] Milton E. de Oliveira Filho; Alfeu J. Sguarezi Filho; Ernesto Ruppert. A Three-phase to three-phase matrix converter prototype. SBA: Controle \& Automação Sociedade Brasileira de Automatica.

Print version ISSN 0103-1759, vol.23, no.3, Campinas, May/June, 2012, https://doi.org/10.1590/S0103-17592012000300001. [10] Gontijo G., Soares M., Tricarico T., Dias R., Aredes M. and Guerrero J. Direct Matrix Converter Topologies with Model Predictive Current Control Applied as Power Interfaces in AC, DC, and Hybrid Microgrids.

University of Rio de Janeiro (COPPE/UFRJ), Rio de Janeiro-RJ 21941-901, Brazil. Published: 27 August 2019.

[11] Cha Han Ju. Dissertation “Analysis and design of matrix converters for adjustable Speed drives and distributed power sources". Submitted to the Office of Graduate Studies of Texas A\&M University in partial fulfillment of the requirements for the degree of $\mathrm{PhD}$. August 2004.

[12] Klumpner C. and Pitic C. Hybrid Matrix Converter Topologies: An Exploration of Benefits. University of Nottingham, School of Electrical and Electronic Engineering, Nottingham,

UNITED KINGDOM, PESC 08, 39th IEEE Power Electronics Specialists Conference, June 15-19, 2008, Greece.

[13] Friedli T., Kolar Johann W. Milestones in Matrix Converter Research. IEEE Journal of Industry Applications, Vol.1 No.1, 2012, pp.2-14. 
[14] Voitovski A.V, Kalinin L.P. [Investigation of phase-shifting transformer operation with circular phase-angle rotation of voltage]. Issledovanie rejima fazoreguliruiuschego transformatora $\mathrm{s}$ krugovim preobrazovaniem fazi napreajenia. Upravleaemie electroperedachi, Kishinev, izdatelistvo "Stiintsa", 1987. (In Russian) device for power systems interconnection]. Transformatornoie ustroistvo dlea sveazi energosistem. Avtorskoie svidetelistvo SSSR no. 1288764. 8.10.1986.(In Russian)

[16] Berlin E.M. [Flexible electrical connections with multiphase key thyristor devices]. Upravleaemie elektricheskie sveazi s mnogofaznimi tiristornokliuchevimi ustroistvami. Izvestia Akademii nauk SSSR, Energetika I transport, No.1, 1988, pp. 29-38.

[17] Mitar Simić, Tomislav B. Šekara, and Srđan Jokić. Model of Three-Limb Three-Phase Transformer Based on Nonlinear Open Circuit Characteristic with Experimental Verification.

Telfor Journal, Vol. 6, No. 1, 2014, pp.42-47.

[18] Zhigang Zhang, Mingrui Mo, Caizhu Wu. Three-phase distribution transformer connections modeling based on matrix operation method by phase-coordinates. Open Access RESEARCH.
[15] Postolaty V.M., Voitovskii A.V. [Transformer (In Russian).

Journal of Wireless Communications and Networking, (2021) 2021:66, 23 pp., https://doi.org/10.1186/s13638-021-01945-z.

[19] Cornelia A. Bulucea, Doru A., Nicola, Nikos E., Mastorakis and Carmen A. Bulucea. Three-phase power transformer modelling in AC/DC traction substations. MATEC Web of Conferences 292, 01006 (2019), CSCC 2019, https://doi.org/10.1051/matecconf/2019292010006.

[20] Oliveira, L. M. R. and Cardoso, A. J. M.: "Modelling and simulation of three-phase power transformers", Proceedings of the 6th International Conference on Modelling and Simulation of

Electrical Machines, Converters and Systems (ELECTRIMACS 99), vol. 2/3, pp. 257-262, Lisbon, Portugal, September 14-16, 1999.

[21] Harshitha G. B., Santhosh D. S., K. Uday Bhargav. Modelling of Three-Phase Transformer in MATLAB/Simulink. International Journal of Innovative Research in Science, Engineering and Technology, India. www.ijirset.com, Vol. 7, Issue 5, May 2018. DOI:10.15680/IJIRSET.2018.0705081, ISSN (Online): 2319-8753, ISSN (Print): 2347-6710.

[22] Grinshtein B.I., Tolstov Iu.G. [Regulation of grid voltage vector phase angle by switching devices convertor]. Regulirovanie fazi vektora napreajenia seti ventilinim fazopovorotnim ustroistvom. Elektrichestvo, №2, 1992. (In Russian).

\section{Сведения об авторах.}

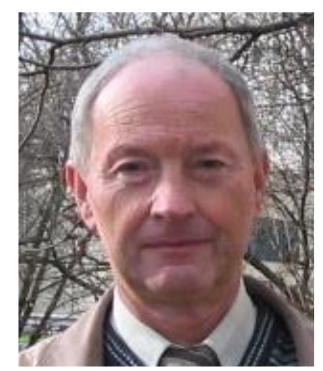

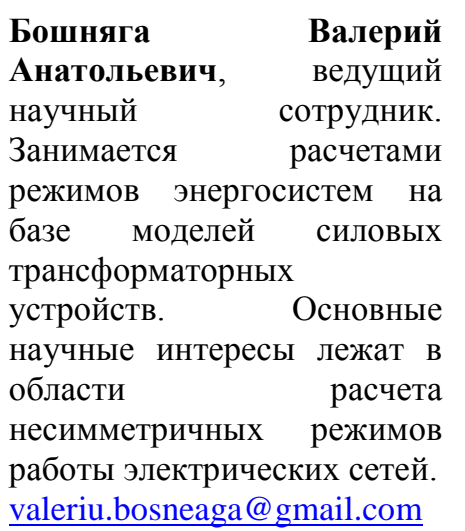

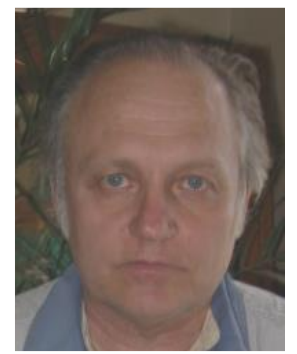

Суслов Виктор Миронович, научный сотрудник, научные интересы связаны с режимами энергетических систем, как установившимися, так и переходными, моделированием элементов энергосистем, в том числе с учетом нелинейности.

svictorm46@gmail.com 